\title{
3-Dimensional kinematics of water/SiO masers in Orion-KL
}

\section{Mi Kyoung KIM, Tomoya Hirota, Kobayashi Hideyuki and VERA project team members}

National Astronomical Observatory of Japan, 2-21-1, Osawa, Mitaka, Tokyo, Japan

\begin{abstract}
Results of multi-epoch VLBI observations toward water/SiO masers in Orion-KL are presented. We conducted high-resolution VLBI observations of water/SiO masers with VERA to probe the structure and the kinematics of the disk/outflow in Orion-KL. The VERA observations provide the positions and proper motions of masers features in Orion-KL with the highest accuracy ever observed. The results of water and $\mathrm{SiO}$ maser observations suggest that Source I is a massive YSO with an accretion disk and a collimated outflow.
\end{abstract}

Keywords. masers, stars:formation

\section{Introduction}

It is known that there are two kind of outflows in Orion-KL: a wide-angle, northwestsoutheast high-velocity outflow, and a northeast-southwest low-velocity outflow. Although the explosive outflows have been studied by a number of observations (see Genzel \& Stutzki (1989) for a review), the origins of outflows and the properties of the driving sources remain debated until now. To investigate the nature of the outflows and its driving source, it is essential to study the 3-dimensional motion of gas associated with the outflows in Orion-KL.

The bright water and $\mathrm{SiO}$ masers are detected in Orion-KL, and the masers are a good tracers of an outflow and a disk surrounding YSOs. Thus, we conducted high-resolution VLBI observations of water and $\mathrm{SiO}$ masers in Orion-KL with VERA to probe the structure and the kinematics of the disk/outflow in this region. $22.2 \mathrm{GHz}$ water masers are observed 6 times during 2005-2006, and simultaneous observations for $v=1,2 j=1-0 \mathrm{SiO}$ masers were done 11 times during 2006-2008. In all observations, Orion-KL and J05410541 were observed simultaneously in dual-beam mode for phase-referencing observation. Typical beam size was $1.7 \times 0.9$ mas for water maser, and $0.8 \times 0.4$ mas for $\mathrm{SiO}$ masers. The velocity resolution for both maser line was $0.21 \mathrm{~km} / \mathrm{s}$.

\section{Results and Discussion}

For both water and $\mathrm{SiO} v=1,2 J=1-0$ masers, we made multi-epoch maps and measured the absolute proper motions of maser features. SiO masers are confined to four arms of $\sim 200 \times 200$ mas in an X-shape distribution, and water masers are distributed in a $\sim 20 \times 20$ arcsec region along the low-velocity outflow in Orion-KL. To obtain the absolute proper motions, we measured the proper motions relative to the reference spot, then we added the absolute proper motion of the reference spot to all measured relative proper motions. For the absolute proper motions of the reference spots, we referred to the values from Hirota et al. (2007) for the water maser and Kim et al. (2008) For the $\mathrm{SiO} v=2 J=1-0$ maser. For the $\mathrm{SiO} v=1 J=1-0$ maser, we measured an absolute proper 


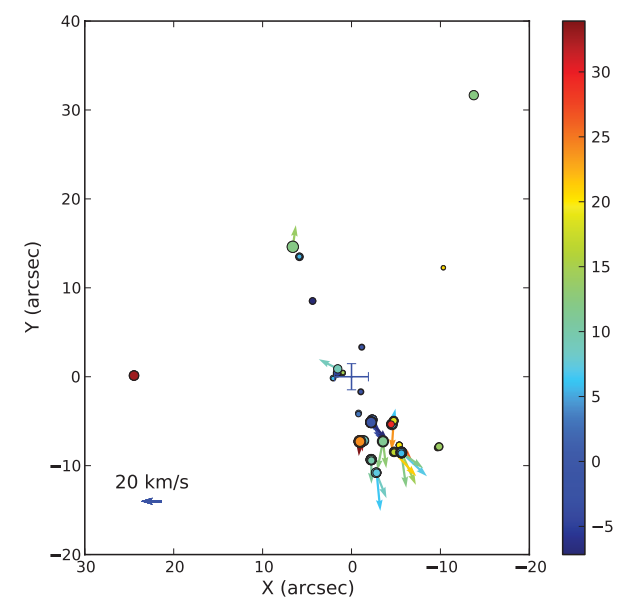

Figure 1. The positions and the absolute proper motions of water masers. The blue cross represents the position of the center from fitting result. The positions of YSOs, $\mathrm{I}, \mathrm{n}$ and $\mathrm{BN}$ are marked by blue dots.

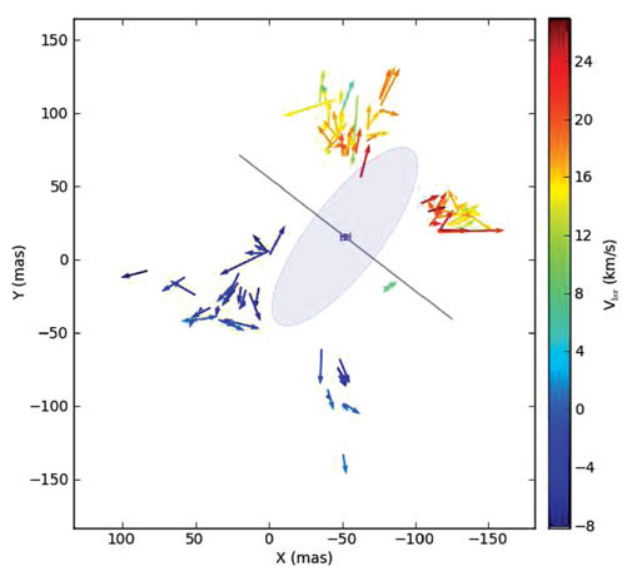

Figure 2. The positions and the internal motions of $\mathrm{SiO}$ masers. The proper motions of Source I is subtracted from the absolute proper motions of each features. The results of fitting are marked: The position of center is marked by the blue cross, the grey line represents the axis of rotation. The aspect ratio of the ellipse is consistent with the inclination of $19.39^{\circ}$.

motion with the annual parallax of 2.39 mas (Kim et al. 2008). The results are shown in Fig. 1, 2.

Then, we fitted the observed data $\left(V_{x, i}, V_{y, i}, V_{z, i}\right),\left(X_{i}, Y_{i}\right)$ to the kinematic models by the least-square fitting method. For the $\mathrm{SiO}$ masers, we supposed an expanding, rotating velocity field $\mathrm{V}_{\mathrm{obs}}=($ Keplerian rotation $)+($ expansion $)+($ systemic motion $)$, because the $\mathrm{V}_{\text {los }}$ gradient of $\sim-0.5 \mathrm{~km} / \mathrm{s} / \mathrm{AU}$ along arms and the existence of system velocity features between arms can be explained by rotating motions (Kim et al. 2008, Matthews et al. 2010). For water masers, we simply suppose that the water masers are moving outward from a center of expansion, $\mathrm{V}_{\mathrm{obs}}=($ expansion $)+($ systemic motion $)$, since their motions show no tendency of acceleration or rotation.

The fitting results show that: 1) the $\mathrm{SiO}$ masers arise from rotating, expanding material around Source I, possibly on the surface of an accretion disk. Its rotating velocity is $\sim 24$ $\mathrm{km} / \mathrm{s}$ at $25 \mathrm{AU}$ from Source I and the estimated central mass is $\mathrm{M} \sim 14 \pm 1 \mathrm{M}_{\odot} .2$ ) the estimated position and proper motion of center of expansion of water masers are consistent with the position and the proper motion of Source I. Water masers trace the outflow from Source I with the expanding velocity of $22 \mathrm{~km} / \mathrm{s}$, which is perpendicular to the disk traced by $\mathrm{SiO}$ masers.

\section{References}

Genzel, R. \& Stutzki, J. 1989, ARAA, 27, 41-85

Hirota, T., et al. 2007, PASJ, 59, 897

Kim, M., et al. 2008, PASJ, 60, 991

Matthews, L. D., et al. 2010, ApJ, 708, 80 